\title{
Embeddings into Orlicz Spaces for Functions from Unbounded Irregular Domains
}

\author{
Petteri Harjulehto $^{1} \cdot$ Ritva Hurri-Syrjänen ${ }^{2}$
}

Received: 13 June 2018 / Accepted: 31 January 2019 / Published online: 9 February 2019

(c) The Author(s) 2019

\section{Abstract}

We study Sobolev functions defined in unbounded irregular domains in the Euclidean $n$-space. We show that there exist embeddings into suitable Orlicz spaces from the space $L_{p}^{1}, 1 \leq p<n$. It turns out that the corresponding Orlicz function depends on the geometry of the domain. The results are sharp for $L_{1}^{1}$-functions.

Keywords Riesz potential · Pointwise estimate - Orlicz space · Unbounded convex domain · Non-smooth domain $\cdot$ Sobolev inequality $\cdot$ Poincaré inequality

Mathematics Subject Classification $31 \mathrm{C} 15 \cdot 42 \mathrm{~B} 20 \cdot 26 \mathrm{D} 10 \cdot 46 \mathrm{E} 30 \cdot 46 \mathrm{E} 35$

\section{Introduction}

In this paper we study inequalities

$$
\inf _{b \in \mathbb{R}}\|u-b\|_{L^{H}(D)} \leq C\|\nabla u\|_{L^{p}(D)},
$$

in unbounded irregular domains $D$ in $\mathbb{R}^{n}$. Here the target space $L^{H}(D)$ is an Orlicz space and it depends on the geometry of $D$. The function $u$ belongs to $L_{p}^{1}(D)=\left\{u \in L_{\text {loc }}^{1}(D):|\nabla u| \in L^{p}(D)\right\}$. Our proof is based on engulfing $D$ by bounded domains $D_{i}$ from inside. Thus we also study bounded domains and calculate

Communicated by Anatoly Golberg, David Shoikhet, Eduard Yakubov and Lawrence Zalcman.

$\bowtie$ Ritva Hurri-Syrjänen

ritva.hurri-syrjanen@helsinki.fi

Petteri Harjulehto

petteri.harjulehto@utu.fi

1 Department of Mathematics and Statistics, University of Turku, 20014 Turku, Finland

2 Department of Mathematics and Statistics, University of Helsinki, 00014 Helsinki, Finland

Birkhäuser 
the constants for the corresponding inequalities so that their constants do not blow up as $\operatorname{diam}\left(D_{i}\right) \rightarrow \infty$.

Although embeddings for functions defined in bounded irregular domains have been studied systematically, see for example $[13,16]$, unbounded irregular domains seem to have been studied less, we refer to [10,13].

A classical example of an embedding into an Orlicz space for Sobolev functions from the Sobolev space $W^{1, n}$ is in [18]. But also, if the domain is irregular then an Orlicz space can be a natural target space for functions defined in $L_{p}^{1}$ as in [6,8]. For papers where an Orlicz space is a target space when the functions come from another Orlicz space we refer to $[3,4]$.

To be more precise, we assume that bounded domains $D_{i}$ are $\varphi$-John domains, that is, every point can be connected to a central point of the domain by a flexible cone of the type $\left\{\left(x, x^{\prime}\right) \in \mathbb{R} \times \mathbb{R}^{n-1}:\left|x^{\prime}\right|<\varphi(x)\right\}$. Here the function $\varphi$ satisfies weak Orlicztype conditions, we refer to Sect. 2. We showed in [7, Theorem 4.4, Theorem 3.5] that every $u \in L_{p}^{1}\left(D_{i}\right)$, can be estimated pointwise almost everywhere by the modified Riesz potential of its gradient

$$
\left|u(x)-u_{D_{i}}\right| \leq C \int_{D_{i}} \frac{|\nabla u(y)|}{\varphi(|x-y|)^{n-1}} d y,
$$

and the modified Riesz potential can be estimated pointwise by the maximal operator

$$
H\left(\int_{G} \frac{|f(y)|}{\varphi(|x-y|)^{n-1}} d y\right) \leq C(M f(x))^{p},
$$

where $H$ is an $N$-function. This is a generalization of Hedberg's method [9, Lemma, Theorem 1]. In the present paper we modify the definition of $\varphi$-John domain so that for $t \geq 1$ the function $\varphi$ grows linearly, we refer to (1.4). This definition keeps the class of uniformly bounded $\varphi$-John domains invariant but makes it possible to control the constants in (1.2) and (1.3) when $\operatorname{diam}\left(D_{i}\right) \rightarrow \infty$. A proper control of the constants is essential, since bounded domains should engulf the given unbounded domain and the required result for the unbounded domain is obtained as a limit of the results to the engulfing bounded domains. Then, we show that $N$-function $H$ can be calculated from the geometry of the domain.

The following theorem tells which kind of $N$-functions we are interested in. These $N$-functions can encode and reveal the geometry of the domain.

Theorem 1.1 Let $1 \leq p<n$. Let the continuous, strictly increasing function $\varphi$ : $[0, \infty) \rightarrow[0, \infty)$ be such that $\varphi(0)=\lim _{t \rightarrow 0^{+}} \varphi(t)=0$ and suppose that $\varphi$ satisfies the $\Delta_{2}$-condition and the inequality $\frac{\varphi\left(t_{1}\right)}{t_{1}} \leq \frac{\varphi\left(t_{2}\right)}{t_{2}}$ whenever $0<t_{1} \leq t_{2}$. Assume that there exists $\alpha \in[1, n /(n-1))$ such that $t^{\alpha} / \varphi(t)$ is increasing for $t>0$. If

$$
\psi(t)= \begin{cases}\varphi(t) & \text { when } 0 \leq t \leq 1 \\ \varphi(1) t & \text { when } t \geq 1\end{cases}
$$


then there exists an $N$-function $H$ that satisfies the $\Delta_{2}$-condition, and

$$
H^{-1}(t) \approx \frac{t^{\frac{1}{p}-1}}{\psi\left(t^{-\frac{1}{n}}\right)^{n-1}} \quad \text { for } t>0
$$

where the implicit constant depends only on $n$ and $p$.

By Theorem 1.1 we prove as an intermediate step the Sobolev-type inequality (1.1) for functions defined in bounded $\varphi$-John domains $D_{i}$, in Theorem $4.1(1<p<n)$ and Theorem $4.2(p=1)$. These results seem to be new and they recover some known results when $p=1$. By using these bounded domains' results we obtain our main result for unbounded domains.

Theorem 1.2 Assume that the function $\varphi$ satisfies the conditions (1)-(5), with $C_{\varphi}=1$ in the condition (4), from the beginning of Sect. 2. Assume that there exists $\alpha \in$ $[1, n /(n-1))$ such that $t^{\alpha} / \varphi(t)$ is increasing for $t>0$. Let the function $\psi$ be defined as in (1.4). Let $D$ in $\mathbb{R}^{n}, n \geq 2$, be an unbounded domain that satisfies the following conditions:

(a) $D=\cup_{i=1}^{\infty} D_{i}$, where $\left|D_{1}\right|>0$;

(b) $\bar{D}_{i} \subset D_{i+1}$ for each $i$;

(c) each $D_{i}$ is a bounded $\varphi$-cigar John domain with a constant $c_{J}$.

Let $1 \leq p<n$. Let $H$ be an $N$-function from Theorem 1.1. Then there exits a constant $C$ such that the inequality

$$
\inf _{b \in \mathbb{R}}\|u-b\|_{L^{H}(D)} \leq C\|\nabla u\|_{L^{p}(D)},
$$

holds for every $u \in L_{p}^{1}(D)$. Here the constant $C$ depends only on $n, p, C_{H}^{\Delta_{2}}, C_{\varphi}^{\Delta_{2}}, c_{J}$, and $\operatorname{diam}\left(D_{1}\right)$.

We give examples in Example 4.5. Finally in Sect. 5 we show that the target space cannot be a Lebesgue space in general.

\section{John Domains}

Throughout the paper we let the function $\varphi:[0, \infty) \rightarrow[0, \infty)$ satisfy the following conditions

(1) $\varphi$ is continuous,

(2) $\varphi$ is strictly increasing,

(3) $\varphi(0)=0$,

(4) there exists a constant $C_{\varphi} \geq 1$ such that

$$
\frac{\varphi\left(t_{1}\right)}{t_{1}} \leq C_{\varphi} \frac{\varphi\left(t_{2}\right)}{t_{2}}
$$

whenever $0<t_{1} \leq t_{2}$, 
(5) $\varphi$ satisfies the $\Delta_{2}$-condition i.e. there exists a constant $C_{\varphi}^{\Delta_{2}} \geq 1$ such that $\varphi(2 t) \leq$ $C_{\varphi}^{\Delta_{2}} \varphi(t)$ for every $t>0$.

We write

$$
\psi(t)= \begin{cases}\varphi(t) & \text { if } 0 \leq t \leq 1 \\ \varphi(1) t & \text { if } t \geq 1\end{cases}
$$

Now, if $\varphi$ satisfies the conditions (1)-(5), then $\psi$ does, too, and the constant in (4) is the same for the functions $\varphi$ and $\psi$, that is $C_{\varphi}=C_{\psi}$.

The definition of a bounded John domain goes back to John [12, Definition, p. 402] who defined an inner radius and an outer radius domain, and later this domain was renamed as a John domain in [14, 2.1].

We extend the definition of John domains following Väisälä [17, 2.1] in the classical case. Let $E$ in $\mathbb{R}^{n}, n \geq 2$, be a closed rectifiable curve with endpoints $a$ and $b$. The subcurve between $x, y \in E$ is denoted by $E[x, y]$. For $x \in E$ we write

$$
q(x)=\min \{\ell(E[a, x]), \ell(E[x, b])\},
$$

where $\ell(E[a, x])$ is the length of the subcurve $E[a, x]$.

Definition 2.1 A bounded or an unbounded domain $D$ in $\mathbb{R}^{n}$ is a $\varphi$-cigar John domain if there exists a constant $c_{J}>0$ such that each pair of points $a, b \in D$ can be joined by a closed rectifiable curve $E$ in $D$ such that

$$
\operatorname{Cig} E(a, b)=\bigcup\left\{B\left(x, \frac{\psi(q(x))}{c_{J}}\right): x \in E \backslash\{a, b\}\right\} \subset D
$$

where $B(x, r)$ is an open ball centered at $x$ with a radius $r>0$ and the function $\psi$ is defined as in (2.1).

The set $\operatorname{Cig} E(a, b)$ is called a cigar with core $E$ joining $a$ and $b$. We point out that if $D$ is a $\varphi$-cigar John domain with $\varphi(t)=t^{p}, p \geq 1$, then it is a $\varphi$-cigar John domain with $\varphi(t)=t^{q}$ for every $q \geq p$. For the case $\psi(t)=\varphi(t)=t$ for all $t \geq 0$, in Definition 2.1, we refer to [17, 2.1] and [15, 2.11 and 2.13]. Note that it is crucial that the length of the curve does not depend on the distance between the end points $a$ and $b$. In bounded uniform domains the length of the cigar depends on $|a-b|$ but they are much more regular than our cigar John domains, see [15].

If $D$ is a bounded domain then the following definition from [7, Definition 4.1] for a $\psi$-John domain gives an equivalent definition to a bounded $\varphi$-cigar John domain.

Definition 2.2 A bounded domain $D$ in $\mathbb{R}^{n}, n \geq 2$, is a $\psi$-John domain if there exist a constants $0<\alpha \leq \beta<\infty$ and a point $x_{0} \in D$ such that each point $x \in D$ can be joined to $x_{0}$ by a rectifiable curve $\gamma:[0, \ell(\gamma)] \rightarrow D$, parametrized by its arc length, such that $\gamma(0)=x, \gamma(\ell(\gamma))=x_{0}, \ell(\gamma) \leq \beta$, and 


$$
\psi(t) \leq \frac{\beta}{\alpha} \operatorname{dist}(\gamma(t), \partial D) \text { for all } t \in[0, \ell(\gamma)]
$$

The point $x_{0}$ is called a John center of $D$ and $\gamma$ is called a John curve of $x$.

Remark 2.3 (1) If the function $\psi$ is defined as in (2.1) with the function $\varphi$, then a bounded domain is a $\psi$-John domain if and only if it is a $\varphi$-John domain.

(2) If $\psi(t)=t$, then our definition for bounded $\psi$-John domains coincides with the definition of the classical John domains. If $\psi(t)=t^{\lambda}, \lambda \geq 1$ then our definition for bounded $\psi$-John domains coincides with the definition of the flexible cone condition in $[2]$.

Theorem 2.4 Let $D$ be a bounded domain. If $D$ is a $\psi$-John domain then $D$ is a $\varphi$ cigar John domain. On the other hand, if $D$ is a $\varphi$-cigar John domain with a constant $c_{J}$, then $D$ is a $\psi$-John domain with constants

$$
\alpha=\frac{\psi\left(\frac{1}{4 c_{J}} \psi\left(\frac{1}{4} \operatorname{diam}(D)\right)\right)}{c_{J} \varphi(1) C_{\varphi}(\varphi(1)+1)}, \quad \beta=\max \left\{2, \alpha, \frac{c_{J} \operatorname{diam}(D)}{\varphi(1)}\right\} .
$$

Note that when $\operatorname{diam}(D) \rightarrow \infty$, then $\alpha \rightarrow \infty$ with the same speed as $\operatorname{diam}(D)$.

Proof Assume first that $D$ is a $\psi$-John domain with a John center $x_{0}$. Let $a, b \in D$ and let the John curves $\gamma_{1}$ and $\gamma_{2}$ connect them to $x_{0}$, respectively. We may assume that $a, b \in D \backslash B\left(x_{0}, \operatorname{dist}\left(x_{0}, \partial D\right)\right)$, since inside the ball the points can be connected by two straight lines going via the center of the ball $B\left(x_{0}\right.$, dist $\left.\left(x_{0}, \partial D\right)\right)$. Let $E$ be a curve from $a$ to $b$ given by $\gamma_{1}$ and $\gamma_{2}$. Then,

$$
\operatorname{Cig} E(a, b)=\bigcup_{t \in\left(0, \ell\left(\gamma_{1}\right)\right]} B\left(\gamma_{1}(t), \frac{\alpha \psi(t)}{\beta}\right) \cup \bigcup_{t \in\left(0, \ell\left(\gamma_{2}\right)\right]} B\left(\gamma_{2}(t), \frac{\alpha \psi(t)}{\beta}\right) \subset D
$$

and thus $D$ is a $\varphi$-cigar John domain.

Assume then that $D$ is a $\varphi$-cigar John domain. Let us carefully choose a suitable John center so that the center is not too close to the boundary of $D$. Let $x, y \in D$ such that $|x-y| \geq \frac{1}{2} \operatorname{diam}(D)$. Let $E$ be a core of a John cigar that connects $x$ and $y$. Then the length of $E$ is at least $\frac{1}{2} \operatorname{diam}(D)$. Let $x_{0}$ be the center of $E$. Then

$$
\operatorname{dist}\left(x_{0}, \partial D\right) \geq \frac{\psi\left(\frac{1}{4} \operatorname{diam}(D)\right)}{c_{J}}
$$

and we choose

$$
r=\frac{\psi\left(\frac{1}{4} \operatorname{diam}(D)\right)}{2 c_{J}}
$$

Hence $B\left(x_{0}, 2 r\right) \subset D$. From now on this $r$ and the point $x_{0}$ are fixed in this proof. 
Fig. 1 The cigar from $a$ to $x_{0}$ (the solid line), the core $E$ (the dotted line) and a new carrot (the dashed line)

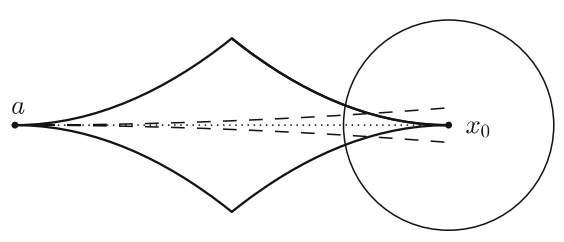

If $a \in B\left(x_{0}, 2 r\right)$, then it can be clearly joint to $x_{0}$ by a line segment and the claim is clear.

For every $a \in D \backslash B\left(x_{0}, 2 r\right)$ there exists a curve $E$ such that the cigar Cig $E\left(a, x_{0}\right) \subset$ $D$ (Fig. 1). Let $\ell(E)$ be the length of $E$, then $\ell(E) \leq 2$ or by Definition 2.1 and (2.1)

$$
\operatorname{diam}(D) \geq 2 \frac{\psi(\ell(E) / 2)}{c_{J}}=2 \frac{\varphi(1) \ell(E)}{2 c_{J}}
$$

i.e. $\ell(E) \leq \max \left\{2, \frac{c_{J} \operatorname{diam}(D)}{\varphi(1)}\right\} \leq \beta$.

Note that the total length of $E$ is at least $2 r$ and the length of $E$ inside the ball $B\left(x_{0}, r\right)$ is at least $r$ and thus for the points in $E \cap \partial B\left(x_{0}, r\right)$ the distance to the boundary is at least $\psi(r / 2) / c_{J}$. Let us choose that

$$
M=\frac{\psi(\beta)}{\psi(r / 2)}=\frac{\varphi(1) \beta}{\psi(r / 2)}
$$

Since $r \leq \ell(E) \leq \beta$ and $\psi$ is increasing, we have $M \geq 1$.

Let $z_{0} \in E$ be the first point from $a$ that satisfies $z_{0} \in \partial B\left(x_{0}, r\right)$. We denote by $\gamma$ the function so that $E$ is parametrized by its arc length such that $\gamma(0)=a, \gamma\left(t_{0}\right)=z_{0}$ and $\gamma(\ell(E))=x_{0}$. We replace $E\left[z_{0}, x_{0}\right]$ by the radius of the ball $B\left(x_{0}, r\right)$, if needed. This new arc is written as $E^{\prime}$. Note that $\ell\left(E^{\prime}\right) \leq \ell(E)$.

Since $M \geq 1$ we have for $t \in\left(0, \frac{1}{2} \ell(E)\right)$ that

$$
\frac{\psi(t)}{M} \leq \psi(t)=\psi(q(\gamma(t)))
$$

By the choice of $M$ in (2.4) we have

$$
\frac{\psi(t)}{M} \leq \psi\left(\frac{r}{2}\right)
$$

for all $t$. On the other hand, for $t \in\left(\frac{1}{2} \ell(E), t_{0}\right)$ the inequality $q(\gamma(t)) \geq r / 2$ holds. Hence, by (2.6)

$$
\frac{\psi(t)}{M} \leq \psi(q(\gamma(t)))
$$


for $t \in\left(\frac{1}{2} \ell(E), t_{0}\right)$, too. These estimates (2.5) and (2.7) give

$$
\bigcup_{t \in\left(0, \ell\left(E^{\prime}\right)\right)} B\left(\gamma(t), \frac{\psi(t)}{M c_{J}}\right) \backslash B\left(x_{0}, r\right) \subset \operatorname{Cig} E\left(a, x_{0}\right) .
$$

By (2.6) we have $\psi(t) \leq M \psi(r / 2)$. By the definition of $\psi$ we have $\psi(r / 2) \leq \varphi(1) r / 2$ if $r \geq 2$, and by condition (4) the inequality $\psi(r / 2) \leq C_{\varphi} \varphi(1) r / 2$ holds if $0<r<2$. Since $C_{\varphi} \geq 1$, we obtain

$$
\psi(t) \leq M \varphi(1) C_{\varphi} r / 2
$$

for all $t \in\left(0, t_{0}\right)$. Since $\varphi(1)$ might be less than one, we estimate

$$
\psi(t) \leq M C_{\varphi}(\varphi(1)+1) r / 2
$$

This inequality and the inclusion $B\left(x_{0}, 2 r\right) \subset D$ yield that

$$
\bigcup_{t \in\left(0, \ell\left(E^{\prime}\right)\right)} B\left(\gamma(t), \frac{\psi(t)}{M C_{\varphi}(\varphi(1)+1) c_{J}}\right) \subset D
$$

Thus, by (2.4)

$\psi(t) \leq M C_{\varphi}(\varphi(1)+1) c_{J} \operatorname{dist}(\gamma(t), \partial D)=\frac{c_{J} \varphi(1) C_{\varphi}(\varphi(1)+1) \beta}{\psi(r / 2)} \operatorname{dist}(\gamma(t), \partial D)$.

This means that we may choose $\alpha=\frac{\psi(r / 2)}{c_{J} \varphi(1) C_{\varphi}(\varphi(1)+1)}$. By using (2.3) we obtain the final $\alpha$. To be sure that $\alpha \leq \beta$ we may choose $\beta$ to be larger if it is necessary. Thus, $D$ is a $\psi$-John domain with $\alpha$ and $\beta$ given in (2.2).

\section{Pointwise Estimates}

We proceed to prove pointwise estimates for domains which are not classical John domains.

We note that by the condition (4) of $\varphi$

$$
\psi(t) \leq C_{\varphi} \varphi(1) t \text { for all } t \geq 0
$$

We recall a covering lemma from [7, Lemma 4.3] which is valid for a bounded $\varphi$-John domain.

Lemma 3.1 [7, Lemma 4.3] Let $\varphi$ satisfy the conditions (1)-(5). Let $\psi:[0, \infty) \rightarrow$ $[0, \infty)$ be defined as in (2.1). Let $D$ in $\mathbb{R}^{n}, n \geq 2$, be a bounded $\psi$-John domain with John constants $\alpha$ and $\beta$. Let $x_{0} \in D$ be the John center. Then for every $x \in$ $D \backslash B\left(x_{0}, \operatorname{dist}\left(x_{0}, \partial D\right)\right)$ there exists a sequence of balls $\left(B\left(x_{i}, r_{i}\right)\right)$ such that $B\left(x_{i}, 2 r_{i}\right)$ 
is in $D$ for each $i=0,1, \ldots$, and for some constants $K=K\left(\alpha, \operatorname{dist}\left(x_{0}, \partial D\right), \beta, \varphi\right)$, $N=N(n)$, and $M=M(n)$

- $B_{0}=B\left(x_{0}, \frac{1}{2} \operatorname{dist}\left(x_{0}, \partial D\right)\right)$;

- $\psi\left(\operatorname{dist}\left(x, B_{i}\right)\right) \leq K r_{i}$, and $r_{i} \rightarrow 0$ as $i \rightarrow \infty$;

- no point of the domain $D$ belongs to more than $N$ balls $B\left(x_{i}, r_{i}\right)$; and

- $\left|B\left(x_{i}, r_{i}\right) \cup B\left(x_{i+1}, r_{i+1}\right)\right| \leq M\left|B\left(x_{i}, r_{i}\right) \cap B\left(x_{i+1}, r_{i+1}\right)\right|$.

Proof The proof is in [7, Lemma 4.3]. We recall only the proof of the inequality $\psi\left(\operatorname{dist}\left(x, B_{i}\right)\right) \leq K r_{i}$, since we have to show that constant $K$ does not blow up when $\operatorname{diam}(D) \rightarrow \infty$.

Let $x \in D \backslash B\left(x_{0}, \operatorname{dist}\left(x_{0}, \partial D\right)\right)$. Let $\gamma$ be a John curve joining $x$ to $x_{0}$, its arc length written as $l$. We write $B_{0}^{\prime}=B\left(x_{0}, \frac{1}{4} \operatorname{dist}\left(x_{0}, \partial D\right)\right)$ and consider the balls $B_{0}^{\prime}$ and

$$
B\left(\gamma(t), \frac{1}{4} \operatorname{dist}(\gamma(t), \partial D \cup\{x\})\right), \quad \text { where } t \in(0, l)
$$

By the Besicovitch covering theorem, there is a sequence of closed balls $\overline{B_{1}^{\prime}}, \overline{B_{2}^{\prime}}, \ldots$ and $\overline{B_{0}^{\prime}}$ that cover the set $\{\gamma(t): t \in[0, l]\} \backslash\{x\}$ and have a uniformly bounded overlap depending on $n$ only. We write $B\left(x_{i}, r_{i}\right)=2 B_{i}^{\prime}$ for every $i=0,1,2, \ldots$, where $x_{i}=\gamma\left(t_{i}\right), t_{i} \in(0, l), r_{0}=\frac{1}{2} \operatorname{dist}\left(x_{0}, \partial D\right)$, and $r_{i}=\frac{1}{2} \operatorname{dist}\left(x_{i}, \partial D \cup\{x\}\right)$.

By the fact that $\varphi$ is an increasing function and by the definition of $\psi$-John domain we obtain

$$
\psi\left(\operatorname{dist}\left(x, B_{0}\right)\right) \leq \psi(l) \leq \psi(\beta) \leq C_{\varphi} \varphi(1) \beta \leq \frac{c \beta r_{0}}{\operatorname{dist}\left(x_{0}, \partial D\right)}
$$

Let us suppose then that $i \geq 1$. If $r_{i}=\frac{1}{2} \operatorname{dist}\left(x_{i}, x\right)$, then by (3.1) we obtain

$$
\psi\left(\operatorname{dist}\left(x, B\left(x_{i}, r_{i}\right)\right)\right) \leq C_{\varphi} \varphi(1) \operatorname{dist}\left(x, B\left(x_{i}, r_{i}\right)\right) \leq 2 C_{\varphi} \varphi(1) r_{i} .
$$

If $r_{i}=\frac{1}{2} \operatorname{dist}\left(x_{i}, \partial D\right)$, then the fact that $\varphi$ is increasing and the definition of a $\psi$-John domain give

$$
\psi\left(\operatorname{dist}\left(x, B\left(x_{i}, r_{i}\right)\right)\right) \leq \psi\left(\operatorname{dist}\left(x, x_{i}\right)\right) \leq \psi\left(t_{i}\right) \leq \frac{\beta}{\alpha} \operatorname{dist}\left(\gamma\left(t_{i}\right), \partial D\right) \leq \frac{2 \beta}{\alpha} r_{i} .
$$

Remark 3.2 (1) The constant $K$ in the previous lemma can be chosen to be $K=$ $\max \left\{\frac{c \beta}{\operatorname{dist}\left(x_{0}, \partial D\right)}, 2 C_{\varphi} \varphi(1), \frac{2 \beta}{\alpha}\right\}$.

(2) If $D$ is a $\varphi$-cigar John domain and the John center has been chosen as in Theorem 2.4, then 


$$
\begin{aligned}
& \frac{\beta}{\operatorname{dist}\left(x_{0}, \partial D\right)} \\
& \leq \frac{\max \left\{2, \frac{\psi\left(\frac{1}{4 c_{J}} \psi\left(\frac{1}{4} \operatorname{diam}(D)\right)\right)}{c_{J} C_{\varphi} \varphi(1)(\varphi(1)+1)}, \frac{c_{J} \operatorname{diam}(D)}{\varphi(1)}\right\}}{\frac{1}{2 c_{J}} \psi\left(\frac{1}{4} \operatorname{diam}(D)\right)} \rightarrow \max \left\{\frac{1}{2 c_{J} C_{\varphi}(\varphi(1)+1)}, \frac{8 c_{J}^{2}}{\varphi(1)^{2}}\right\}
\end{aligned}
$$

and

$$
\frac{\beta}{\alpha}=\frac{\max \left\{2, \frac{\psi\left(\frac{1}{4 c_{J}} \psi\left(\frac{1}{4} \operatorname{diam}(D)\right)\right)}{{ }_{{ }_{J}} C_{\varphi} \varphi(1)(\varphi(1)+1)}, \frac{c_{J} \operatorname{diam}(D)}{\varphi(1)}\right\}}{\frac{\psi\left(\frac{1}{{ }^{c_{J}} \psi} \psi\left(\frac{1}{4} \operatorname{diam}(D)\right)\right)}{c_{J} C_{\varphi} \varphi(1)(\varphi(1)+1)}} \rightarrow \max \left\{1, \frac{16 c_{J}^{3} C_{\varphi}(\varphi(1)+1)}{\varphi(1)^{2}}\right\}
$$

as $\operatorname{diam}(D) \rightarrow \infty$

We recall the following definitions. Let $G$ be an open set of $\mathbb{R}^{n}$. We denote the Lebesgue space by $L^{p}(G), 1 \leq p<\infty$. By $L_{p}^{1}(G), 1 \leq p<\infty$, we denote those locally integrable functions whose first weak distributional derivatives belongs to $L^{p}(G)$, that is, $L_{p}^{1}(G)=\left\{u \in L_{\mathrm{loc}}^{1}(G):|\nabla u| \in L^{p}(G)\right\}$. By $W^{1, p}(G), 1 \leq p<\infty$, we denote those functions from $L^{p}(G)$ whose first weak distributional derivatives belongs to $L^{p}(G)$, that is, $W^{1, p}(G)=\left\{u \in L^{p}(G):|\nabla u| \in L^{p}(G)\right\}$.

Theorem 2.4 and Lemma 3.1 give the following pointwise estimate which we recall from [7, Theorem 4.4].

Theorem 3.3 Let $\varphi$ satisfy the conditions $(1)-(5)$. Let $\psi:[0, \infty) \rightarrow[0, \infty)$ be as defined in (2.1). Let $D$ in $\mathbb{R}^{n}, n \geq 2$, be a bounded $\varphi$-cigar John domain with a John constant $c_{J}$. Then there exists a finite constant $C$ and $x_{0} \in D$ such that for every $u \in L_{1}^{1}(D)$ and for almost every $x \in D$ the inequality

$$
\left|u(x)-u_{B\left(x_{0}, \operatorname{dist}\left(x_{0}, \partial D\right)\right)}\right| \leq C \int_{D} \frac{|\nabla u(y)|}{\psi(|x-y|)^{n-1}} d y
$$

holds. Here $C=c\left(n, c_{J}, C_{\varphi}, C_{\varphi}^{\Delta_{2}}, \varphi(1), \min \{\operatorname{diam}(D), 1\}\right)$.

We recall the definitions of $N$-functions and Orlicz spaces.

Definition 3.4 A function $H:[0, \infty) \rightarrow[0, \infty)$ is an $N$-function if

(N1) $H$ is continuous,

(N2) $H$ is convex,

(N3) $\lim _{t \rightarrow 0^{+}} \frac{H(t)}{t}=0$ and $\lim _{t \rightarrow \infty} \frac{H(t)}{t}=\infty$. 
Continuity and $\lim _{t \rightarrow 0^{+}} \frac{H(t)}{t}=0$ yield that $H(0)=0$.

Convexity yields that $\frac{H(t)}{t} \leq \frac{H(s)}{s}$ for $0<t<s$ and thus $H$ is a strictly increasing function.

By the notation $f \lesssim g$ we mean that there exists a constant $C>0$ such that $f(x) \leq C g(x)$ for all $x$. The notation $f \approx g$ means that $f \lesssim g \lesssim f$.

Two $N$-functions $H$ and $K$ are equivalent, which is written as $H \simeq K$, if there exists $m \geq 1$ such that $H(t / m) \leq K(t) \leq H(m t)$ for all $t>0$. Equivalent $N$-functions give the same space with comparable norms. We point out that $H \simeq K$ if and only if for the inverse functions $H^{-1} \approx K^{-1}$.

We assume that $H$ satisfies the $\Delta_{2}$-condition, that is, there exists a constant $C_{H}^{\Delta_{2}}$ such that

$$
H(2 t) \leq C_{H}^{\Delta_{2}} H(t) \text { for all } t>0 .
$$

The constant $C_{H}^{\Delta_{2}}$ is called the $\Delta_{2}$-constant of $H$.

Let $G$ in $\mathbb{R}^{n}$ be an open set.

We study the Orlicz space $L^{H}(G)$ which means the space of all measurable functions $u$ defined on $G$ such that

$$
\int_{G} H(\lambda|u(x)|) d x<\infty
$$

for some $\lambda>0$.

The Orlicz space $L^{H}(G)$ equipped with the Luxemburg norm

$$
\|u\|_{L^{\Phi}(G)}=\inf \left\{\lambda>0: \int_{G} \Phi\left(\frac{|u(x)|}{\lambda}\right) d x \leq 1\right\}
$$

is a Banach space.

Let $G$ in $\mathbb{R}^{n}$ be an open set. Assume that $f \in L^{1}(G)$. The centered HardyLittlewood maximal operator is defined as

$$
M f(x)=\sup _{r>0} f_{B(x, r)}\left|f(y) \chi_{G}(x)\right| d x,
$$

where the function $f \chi_{G}$ is understood to be zero in the complement of $G$. We recall the following theorem from [7, Theorem 3.5] which is applied to the function $f \chi_{G}$.

Theorem 3.5 Let $\varphi$ satisfy the conditions (1)-(5). Let $\psi:[0, \infty) \rightarrow[0, \infty)$ be defined as in (2.1). Let $1 \leq p<n$ be given. Suppose that there exists a continuous function $h:[0, \infty) \rightarrow[0, \infty)$ such that

$$
\sum_{k=1}^{\infty} \frac{\left(2^{-k} t\right)^{n}}{\psi\left(t 2^{-k}\right)^{n-1}} \leq h(t) \text { for all } t>0
$$


Let $\delta:(0, \infty) \rightarrow[0, \infty)$ be a continuous function and let $H:[0, \infty) \rightarrow[0, \infty)$ be an $N$-function satisfying the $\Delta_{2}$-condition. Suppose that there exists a finite constant $C_{H}$ such that the inequality

$$
H\left(h(\delta(t)) t+\psi(\delta(t))^{1-n}(\delta(t))^{n\left(1-\frac{1}{p}\right)}\right) \leq C_{H} t^{p}
$$

holds for all $t>0$. Let $G$ in $\mathbb{R}^{n}$ be an open set. If $\|f\|_{L^{p}(G)} \leq 1$, then there exists a constant $C$ such that the inequality

$$
H\left(\int_{G} \frac{|f(y)|}{\psi(|x-y|)^{n-1}} d y\right) \leq C(M f(x))^{p}
$$

holds for every $x \in G$. Here the constant $C$ depends on $n, p, C_{\varphi}, C_{H}$, and the $\Delta_{2}$-constants of $\varphi$ and $H$ only.

Our goal is to find a formula which would give all suitable functions $H$. Examples of some of these functions were given in [7, Section 6].

Here we do the preparations to find $H$. Assume that there exists $\alpha \in[1, n /(n-1))$ such that $t^{\alpha} / \varphi(t)$ is increasing for $t>0$. This yields that $t^{\alpha} / \psi(t)$ is increasing, too. Under this condition inequality (3.3) holds: Since

$$
\begin{aligned}
\frac{\left(2^{-k} t\right)^{n}}{\psi\left(t 2^{-k}\right)^{n-1}} & =\frac{\left(2^{-k} t\right)^{n}}{\left(2^{-k} t\right)^{\alpha(n-1)}} \cdot \frac{\left(2^{-k} t\right)^{\alpha(n-1)}}{\psi\left(t 2^{-k}\right)^{n-1}} \\
& \leq\left(2^{-k} t\right)^{n-\alpha(n-1)} \frac{t^{\alpha(n-1)}}{\psi(t)^{n-1}}=2^{-k(n-\alpha(n-1))} \frac{t^{n}}{\psi(t)^{n-1}}
\end{aligned}
$$

we have

$$
\sum_{k=1}^{\infty} \frac{\left(2^{-k} t\right)^{n}}{\psi\left(t 2^{-k}\right)^{n-1}} \leq C(n, \alpha) \frac{t^{n}}{\psi(t)^{n-1}}, \quad \text { where } \quad C(n, \alpha)=\frac{2^{\alpha(n-1)}}{2^{n}-2^{\alpha(n-1)}}
$$

Let us define the functions $h$ and $\delta$ such that

$$
h(t)=C(n, \alpha) \frac{t^{n}}{\psi(t)^{n-1}} \quad \text { and } \quad \delta(t)=t^{-\frac{p}{n}} \text { for all } t>0
$$

Then,

$$
\begin{gathered}
h(\delta(t)) t+\psi(\delta(t))^{1-n}(\delta(t))^{n\left(1-\frac{1}{p}\right)}=h\left(t^{-\frac{p}{n}}\right) t+\psi\left(t^{-\frac{p}{n}}\right)^{1-n}\left(t^{-\frac{p}{n}}\right)^{n\left(1-\frac{1}{p}\right)} \\
=\frac{C(n, \alpha) t^{-p}}{\psi\left(t^{-\frac{p}{n}}\right)^{n-1}} t+\frac{t^{1-p}}{\psi\left(t^{-\frac{p}{n}}\right)^{n-1}}=\frac{(C(n, \alpha)+1) t^{1-p}}{\psi\left(t^{-\frac{p}{n}}\right)^{n-1}} .
\end{gathered}
$$


Fig. 2 The function $F$ is not necessary convex

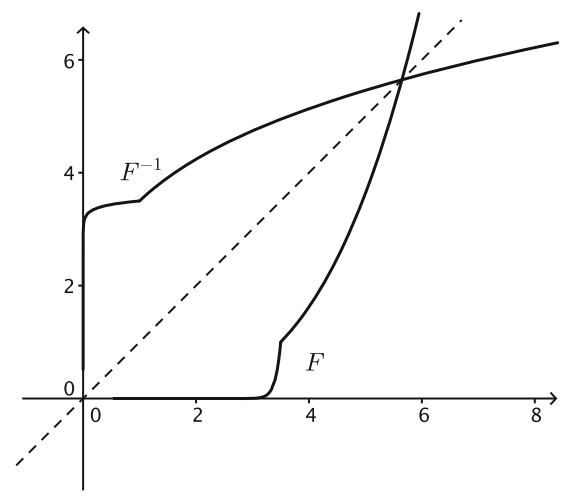

If we choose

$$
F^{-1}(t)=\frac{(C(n, \alpha)+1)\left(t^{1 / p}\right)^{1-p}}{\psi\left(\left(t^{1 / p}\right)^{-\frac{p}{n}}\right)^{n-1}}=\frac{(C(n, \alpha)+1) t^{\frac{1}{p}-1}}{\psi\left(t^{-\frac{1}{n}}\right)^{n-1}}
$$

and assume that the inverse function of $F^{-1}$ exists, that is $\left(F^{-1}\right)^{-1}=: F$ exists, then

$$
h(\delta(t)) t+\psi(\delta(t))^{1-n}(\delta(t))^{n\left(1-\frac{1}{p}\right)}=F^{-1}\left(t^{p}\right)
$$

and thus

$$
F\left(h(\delta(t)) t+\psi(\delta(t))^{1-n}(\delta(t))^{n\left(1-\frac{1}{p}\right)}\right)=F\left(F^{-1}\left(t^{p}\right)\right)=t^{p}
$$

Unfortunately, there is a problem with this function $F$ to be a suitable function $H$; namely, the function $F$ is not necessary convex. For example, if $n=2, \varphi(t)=t^{\frac{3}{2}}$, and $p=1.9$, then the function $F$ is not convex, see Fig. 2. The angle at the point $\left(1, F^{-1}(1)\right)$ comes from the angle of $\psi$ at the point $(1, \psi(1))$. Our main theorem, Theorem 1.1 in Introduction, corrects this point: we show that there exists an $N$ function $H$ that is equivalent with $F$.

Proof of Theorem 1.1 Let us write that

$$
F^{-1}(t)=\frac{t^{\frac{1}{p}-1}}{\psi\left(t^{-\frac{1}{n}}\right)^{n-1}}
$$

for $t>0$ and $F^{-1}(0)=0$. Let us first show that $F^{-1}$ is strictly increasing. We recall that if $\varphi$ satisfies condition (4), then $\psi$ does too, and the constant is the same for both functions. We have 


$$
F^{-1}(t)=t^{\frac{1}{p}-1+\frac{n-1}{n}}\left(\frac{\left(t^{-\frac{1}{n}}\right)}{\psi\left(t^{-\frac{1}{n}}\right)}\right)^{n-1}=t^{\frac{1}{p}-\frac{1}{n}}\left(\frac{\left(t^{-\frac{1}{n}}\right)}{\psi\left(t^{-\frac{1}{n}}\right)}\right)^{n-1}
$$

Since $p<n$ the function $t \mapsto t^{\frac{1}{p}-\frac{1}{n}}$ is strictly increasing. Since the function $t \mapsto t^{-\frac{1}{n}}$ is strictly decreasing, condition (4) with $C_{\varphi}=1$ yields that $t \mapsto\left(t^{-\frac{1}{n}}\right) / \psi\left(t^{-1 / n}\right)$ is strictly increasing. These together yield that $F^{-1}$ is strictly increasing.

This yields that the function $F$ exists and is strictly increasing.

Let us show that $\lim _{t \rightarrow 0^{+}} F^{-1}(t)=0$. Since $p<n$ we obtain

$$
\lim _{t \rightarrow 0^{+}} F^{-1}(t)=\lim _{t \rightarrow 0^{+}} \frac{t^{\frac{1}{p}-1}}{\psi\left(t^{-\frac{1}{n}}\right)^{n-1}}=\lim _{t \rightarrow 0^{+}} \varphi(1)^{1-n} t^{\frac{n-1}{n}+\frac{1}{p}-1}=0 .
$$

Let us show that $\lim _{t \rightarrow \infty} F^{-1}(t)=\infty$. Since $t / \varphi(t)$ is decreasing, by the condition (4), and by $p<n$ we obtain

$$
\lim _{t \rightarrow \infty} F^{-1}(t)=\lim _{t \rightarrow \infty} \frac{t^{\frac{1}{p}-1}}{\psi\left(t^{-\frac{1}{n}}\right)^{n-1}}=\lim _{t \rightarrow \infty} t^{\frac{1}{p}-\frac{1}{n}}\left(\frac{t^{-\frac{1}{n}}}{\psi\left(t^{-\frac{1}{n}}\right)}\right)^{n-1} \geq \lim _{t \rightarrow \infty} \frac{t^{\frac{1}{p}-\frac{1}{n}}}{\varphi(1)^{n-1}}=\infty
$$

We have shown that $F^{-1}:[0, \infty) \rightarrow[0, \infty)$ is bijective.

Let us then study the condition

$$
\frac{F(s)}{s}<\frac{F(t)}{t} \text { for } 0<s<t
$$

Since $F^{-1}$ is a strictly increasing bijection, inequality (3.6) is equivalent to

$$
\frac{s}{F^{-1}(s)}<\frac{t}{F^{-1}(t)} \text {. }
$$

Since $t^{\alpha} / \varphi(t)$ is increasing, then $\varphi(t) / t^{\alpha}$ is decreasing and $\psi(t) / t^{\alpha}$ is decreasing, too. We note that $1-\frac{\alpha(n-1)}{n}>0$, since $\alpha<\frac{n}{n-1}$. We obtain

$$
\begin{aligned}
\frac{s}{F^{-1}(s)} & =s^{2-\frac{1}{p}} \psi\left(s^{-\frac{1}{n}}\right)^{n-1}=s^{2-\frac{1}{p}-\frac{\alpha(n-1)}{n}}\left(\frac{\psi\left(s^{-\frac{1}{n}}\right)}{\left(s^{-\frac{1}{n}}\right)^{\alpha}}\right)^{n-1} \\
& =s^{1-\frac{1}{p}+1-\frac{\alpha(n-1)}{n}}\left(\frac{\psi\left(s^{-\frac{1}{n}}\right)}{\left(s^{-\frac{1}{n}}\right)^{\alpha}}\right)^{n-1}<t^{1-\frac{1}{p}+1-\frac{\alpha(n-1)}{n}}\left(\frac{\psi\left(t^{-\frac{1}{n}}\right)}{\left(t^{-\frac{1}{n}}\right)^{\alpha}}\right)^{n-1}=\frac{t}{F^{-1}(t)}
\end{aligned}
$$

and thus inequality (3.6) holds. 
Let us then show that $F^{-1}(c s) \geq 2 F^{-1}(s)$ for all $s \geq 0$ with $c=2^{\frac{n p}{n-p}}$. The inequality $F^{-1}(c s) \geq 2 F^{-1}(s)$ is equivalent to

$$
2 \frac{\psi\left(\left(\frac{1}{c s}\right)^{\frac{1}{n}}\right)^{n-1}}{\left(\frac{1}{c s}\right)^{1-\frac{1}{p}}} \leq \frac{\psi\left(\left(\frac{1}{s}\right)^{\frac{1}{n}}\right)^{n-1}}{\left(\frac{1}{s}\right)^{1-\frac{1}{p}}}
$$

By the condition (4) of $\varphi$ and the inequality $p<n$, we obtain

$$
\begin{aligned}
2 \frac{\psi\left(\left(\frac{1}{c s}\right)^{\frac{1}{n}}\right)^{n-1}}{\left(\frac{1}{c s}\right)^{1-\frac{1}{p}}} & =2\left(\frac{\psi\left(\left(\frac{1}{c s}\right)^{\frac{1}{n}}\right)}{\left(\frac{1}{c s}\right)^{\frac{1}{n}}}\right)^{n-1}\left(\frac{1}{c s}\right)^{\frac{n-1}{n}-1+\frac{1}{p}} \\
& =\left(\frac{\psi\left(\left(\frac{1}{c s}\right)^{\frac{1}{n}}\right)}{\left(\frac{1}{c s}\right)^{\frac{1}{n}}}\right)^{n-1}\left(\frac{1}{s}\right)^{\frac{n-1}{n}-1+\frac{1}{p}} \\
& \leq\left(\frac{\psi\left(\left(\frac{1}{s}\right)^{\frac{1}{n}}\right)}{\left(\frac{1}{s}\right)^{\frac{1}{n}}}\right)^{n-1}\left(\frac{1}{s}\right)^{\frac{n-1}{n}-1+\frac{1}{p}}=\frac{\psi\left(\left(\frac{1}{s}\right)^{\frac{1}{n}}\right)^{n-1}}{\left(\frac{1}{s}\right)^{1-\frac{1}{p}}}
\end{aligned}
$$

The inequality $F^{-1}(c s) \geq 2 F^{-1}(s)$ yields that $F$ satisfies the $\Delta_{2}$-condition. Let us write $F(t)=s$. Then $F^{-1}(s)=t$. Since $F$ is increasing, we have

$$
F(2 t)=F\left(2 F^{-1}(s)\right) \leq F\left(F^{-1}(c s)\right)=c s=c F(t) .
$$

Since $F$ satisfies $\Delta_{2}$-condition it is finite everywhere and hence (3.6) yields that $F(0)=\lim _{s \rightarrow 0^{+}} F(s)=0$ and $\lim _{s \rightarrow \infty} F(s)=\infty$. Since $\psi$ is continuous, we find that $F^{-1}$ is continuous on $(0, \infty)$ and hence also $F$ is continuous on $(0, \infty)$ and moreover on $[0, \infty)$.

Hästö has shown in [11, Proposition 3.1] that if $f:[0, \infty) \rightarrow[0, \infty)$ is leftcontinuous, $f(0)=\lim _{s \rightarrow 0^{+}} f(s)=0, \lim _{s \rightarrow \infty} f(s)=\infty$ and $x \mapsto f(x) / x$ is increasing, then $f$ is equivalent to a convex function. We obtain that $F$ is equivalent to a convex function $H$. Here the implicit constant depends only on the constant in the $\Delta_{2}$-condition, that is, it depends only on $n$ and $p$.

Using $\lim _{t \rightarrow 0^{+}} F^{-1}(t)=0$ and the bijectivity, we obtain

$$
\lim _{t \rightarrow 0^{+}} \frac{F(t)}{t}=\lim _{t \rightarrow 0^{+}} \frac{t}{F^{-1}(t)}=\lim _{t \rightarrow 0^{+}} \frac{t \psi\left(\left(\frac{1}{t}\right)^{\frac{1}{n}}\right)^{n-1}}{\left(\frac{1}{t}\right)^{1-\frac{1}{p}}}=\lim _{t \rightarrow 0^{+}} \varphi(1)^{n-1} t^{1-\frac{1}{p}+1-\frac{n-1}{n}}=0
$$


and thus also $\lim _{t \rightarrow 0^{+}} \frac{H(t)}{t}=0$. This gives that $H$ is right continuous at the origin. Since $F$ satisfies $\Delta_{2}$-condition so does $H$ and thus it is finite everywhere. Thus by convexity the function $H$ is continuous on $[0, \infty)$.

Since $\varphi(t) / t^{\alpha}$ is decreasing and $\alpha<\frac{n}{n-1}$, we obtain

$$
\begin{aligned}
\lim _{t \rightarrow \infty} \frac{F(t)}{t} & =\lim _{t \rightarrow \infty} \frac{t}{F^{-1}(t)}=\lim _{t \rightarrow \infty} t^{2-\frac{1}{p}} \varphi\left(t^{-\frac{1}{n}}\right)^{n-1} \\
& =\lim _{t \rightarrow \infty} t^{2-\frac{1}{p}-\frac{\alpha(n-1)}{n}}\left(\frac{\varphi\left(t^{-\frac{1}{n}}\right)}{\left(t^{-\frac{1}{n}}\right)^{\alpha}}\right)^{n-1} \geq \lim _{t \rightarrow \infty} t^{1-\frac{1}{p}+1-\frac{\alpha(n-1)}{n}}\left(\frac{\varphi(1)}{1^{\alpha}}\right)^{n-1} \\
& =\infty
\end{aligned}
$$

Since the functions $F$ and $H$ are equivalent, this yields that $\lim _{t \rightarrow \infty} \frac{H(t)}{t}=\infty$. Thus we have shown that the function $H$ satisfies the conditions (N1)-(N3).

Remark 3.6 Later it is crucial that

$$
H^{-1}(t) \approx \frac{t^{\frac{1}{p}-1}}{\psi\left(t^{-\frac{1}{n}}\right)^{n-1}}=\frac{t^{\frac{1}{p}-1}}{\varphi(1)^{n-1}\left(t^{-\frac{1}{n}}\right)^{n-1}}=\varphi(1)^{1-n} t^{\frac{n-p}{n p}}
$$

for $0<t \leq 1$. Namely, for every $\varphi$ the function $H$ satisfies $H(t) \approx t^{\frac{n p}{n-p}}$ whenever $0<t \leq 1$.

Example 3.7 Functions $\varphi(t)=t^{\alpha} / \log ^{\beta}(e+1 / t), \alpha \in\left[1, \frac{n}{n-1}\right)$ and $\beta \geq 0$, satisfy the assumptions of Theorem 1.1.

Theorems 1.1 and 3.5 yield the following result.

Theorem 3.8 Let $D$ be an unbounded or a bounded domain in $\mathbb{R}^{n}, n \geq 2$. Let $1 \leq$ $p<n$. If $H$ is the function from Theorem 1.1 and $\|f\|_{L^{p}(D)} \leq 1$, then there exists $a$ constant $C$ such that the pointwise estimate

$$
H\left(\int_{D} \frac{|f(y)|}{\psi(|x-y|)^{n-1}} d y\right) \leq C(M f(x))^{p}
$$

holds for every $x \in D$. Here, $M f$ is the Hardy-Littlewood maximal operator of $f$ and the constant $C$ depends on $n, p$, and the $\Delta_{2}$-constant of $H$ only.

As a corollary we obtain from Theorems 3.3 and 3.8:

Corollary 3.9 Let $1 \leq p<n$. Let the function $H$ be as in Theorem 1.1. If $D$ is a bounded $\varphi$-cigar John domain with a constant $c_{J}$, then there exit a constant $C$ and $a$ point $x_{0} \in D$ such that the pointwise estimate

$$
H\left(\left|u(x)-u_{B\left(x_{0}, \operatorname{dist}\left(x_{0}, \partial D\right)\right)}\right|\right) \leq C(M|\nabla u|(x))^{p}
$$


holds for all $u \in L_{p}^{1}(D)$ with $\|\nabla u\|_{L^{p}(D)} \leq 1$ and for almost every $x \in D$. Here the constant $C$ depends on $n, p, C_{H}, C_{H}^{\Delta_{2}}, C_{\varphi}^{\Delta_{2}}, c_{J}, \varphi(1)$ and $\min \{\operatorname{diam}(D), 1\}$ only.

\section{On Embeddings}

Corollary 3.9 is essential in the proofs of the following Theorems 4.1 and 4.2.

Theorem 4.1 (Bounded domain, $1<p<n$ ) Assume that $\varphi$ satisfies the conditions (1)-(5), $C_{\varphi}=1$ in the condition (4), and there exists $\alpha \in[1, n /(n-1))$ such that $t^{\alpha} / \varphi(t)$ is increasing for $t>0$. Let $\psi$ be defined as in (2.1). Let $D \subset \mathbb{R}^{n}, n \geq 2$, be a bounded $\varphi$-cigar John domain with a constant $c_{J}$. Let $1<p<n$. Then there exists an $N$-function $H$, that satisfies $\Delta_{2}$-condition and

$$
H^{-1}(t) \approx \frac{t^{\frac{1}{p}-1}}{\psi\left(t^{-\frac{1}{n}}\right)^{n-1}} \text { for all } t>0
$$

and there exists a constant $C<\infty$ such that the inequality

$$
\left\|u-u_{D}\right\|_{L^{H}(D)} \leq C\|\nabla u\|_{L^{p}(D)},
$$

holds for every $u \in L_{p}^{1}(D)$. Here the constant $C$ depends on $n, p, C_{H}^{\Delta_{2}}, C_{\varphi}^{\Delta_{2}}, c_{J}$ and $\min \{\operatorname{diam}(D), 1\}$ only.

Proof Theorem 2.4 implies that $D$ is a bounded $\psi$-John domain. Let $x_{0}$ be a John center. Let us denote $B=B\left(x_{0}, \operatorname{dist}\left(x_{0}, \partial D\right)\right)$. Assume that $\|\nabla u\|_{L^{p}(D)} \leq 1$. Corollary 3.9 yields that $H\left(\left|u(x)-u_{B}\right|\right) \leq C(M|\nabla u|(x))^{p}$, where the constant $C$ depends on $n$, $p, C_{H}^{\Delta_{2}}, C_{\varphi}^{\Delta_{2}}, c_{J}$, and $\min \{1, \operatorname{diam}(D)\}$ only. By integrating over $D$ and using the fact that the maximal operator is bounded whenever $1<p<n$, we obtain that

$$
\int_{D} H\left(\left|u(x)-u_{B}\right|\right) d x \leq C \int_{D}(M|\nabla u|(x))^{p} d x \leq C \int_{D}|\nabla u(x)|^{p} d x \leq C .
$$

This yields that the inequality $\left\|u-u_{B}\right\|_{L^{H}(D)} \leq C$ holds for every $u \in L_{p}^{1}(D)$ with $\|\nabla u\|_{L^{p}(D)} \leq 1$. If $\|\nabla u\|_{L^{p}(D)}=0$ then the function is a constant function and the claim holds. Otherwise we apply this inequality to the function $u /\|\nabla u\|_{L^{p}(D)}$ and obtain that $\left\|u-u_{B}\right\|_{L^{H}(D)} \leq C\|\nabla u\|_{L^{p}(D)}$.

We may assume w.l.o.g. that $\|\nabla u\|_{L^{p}(D)} \neq 0$. By the triangle inequality $\| u-$ $u_{D}\left\|_{L^{H}(D)} \leq\right\| u-u_{B}\left\|_{L^{H}(D)}+\right\| u_{B}-u_{D} \|_{L^{H}(D)}$. Here,

$$
\begin{aligned}
\left\|u_{B}-u_{D}\right\|_{L^{H}(D)} & =\left|u_{B}-u_{D}\right|\|1\|_{L^{H}(D)} \leq \frac{\|1\|_{L^{H}(D)}}{|D|}\left\|u-u_{B}\right\|_{L^{1}(D)} \\
& \leq C \frac{\|1\|_{L^{H}(D)}\|1\|_{L^{H^{*}}(D)}}{|D|}\left\|u-u_{B}\right\|_{L^{H}(D)}
\end{aligned}
$$


where $H^{*}$ is the conjugate function of $H$ and $C$ is the constant in Hölder's inequality. It is well known that $\|1\|_{L^{H}(D)}\|1\|_{L^{H^{*}(D)}} \approx|D|$ see [1, Chapter 2, Theorem 5.2]. Hence, we have shown that $\left\|u-u_{D}\right\|_{L^{H}(D)} \leq C\|\nabla u\|_{L^{p}(D)}$ for every $u \in L_{p}^{1}(D)$.

Theorem 4.2 (Bounded domain, $p=1)$ Assume that the function $\varphi$ satisfies the conditions (1)- (5), $C_{\varphi}=1$ in the condition (4), and there exists $\alpha \in[1, n /(n-1))$ such that $t^{\alpha} / \varphi(t)$ is increasing for $t>0$. Let $\psi$ be defined as in (2.1) Let $D \subset \mathbb{R}^{n}$, $n \geq 2$, be a bounded $\varphi$-cigar John domain with a constant $c_{J}$. Then there exists an $N$-function $H$, that satisfies $\Delta_{2}$-condition and

$$
H^{-1}(t) \approx \frac{1}{\psi\left(t^{-\frac{1}{n}}\right)^{n-1}} \quad \text { for all } t>0
$$

such that the inequality

$$
\left\|u-u_{D}\right\|_{L^{H}(D)} \leq C\|\nabla u\|_{L^{1}(D)},
$$

holds for some constant $C$ and for every $u \in L_{p}^{1}(D)$. Here the constant $C$ depends only on $n, C_{H}^{\Delta_{2}}, C_{\varphi}^{\Delta_{2}}, c_{J}$, and $\min \{1, \operatorname{diam}(D)\}$.

The term $\min \{1, \operatorname{diam}(D)\}$ means that the constant depends on the diameter only for small diameters. For large diameters the constant is independent of the diameter.

Proof Let us consider functions $u \in L_{1}^{1}(D)$ such that $\|\nabla u\|_{L^{1}(D)} \leq 1$. The center ball $B\left(x_{0}, \operatorname{dist}\left(x_{0}, \partial D\right)\right)$ is written as $B$. In the proof of Theorem 2.4 we had chosen $x_{0}$ so that $\operatorname{dist}\left(x_{0}, \partial D\right) \geq \psi\left(\frac{1}{4} \operatorname{diam}(D)\right) / c_{J}$. We show that there exists a constant $C<\infty$ such that the inequality

$$
\int_{D} H\left(\left|u(x)-u_{B}\right|\right) d x \leq C
$$

holds whenever $\|\nabla u\|_{L^{1}(D)} \leq 1$. This yields the claim as in the proof of Theorem 4.1.

Since $H$ is increasing, we first estimate

$$
\int_{D} H\left(\left|u(x)-u_{B}\right|\right) d x \leq \sum_{j \in \mathbb{Z}} \int_{\left\{x \in D: 2^{j}<\left|u(x)-u_{B}\right| \leq 2^{j+1}\right\}} H\left(2^{j+1}\right) d x .
$$

Let us define $v_{j}(x)=\max \left\{0, \min \left\{\left|u(x)-u_{B}\right|-2^{j}, 2^{j}\right\}\right\}$ for all $x \in D$. If $x \in$ $\left\{x \in D: 2^{j}<\left|u(x)-u_{B}\right| \leq 2^{j+1}\right\}$, then $v_{j-1}(x) \geq 2^{j-1}$. We obtain

$$
\int_{D} H\left(\left|u(x)-u_{B}\right|\right) d x \leq \sum_{j \in \mathbb{Z}} \int_{\left\{x \in D: v_{j}(x) \geq 2^{j}\right\}} H\left(2^{j+2}\right) d x .
$$


By the triangle inequality we have

$$
v_{j}(x)=\left|v_{j}(x)-\left(v_{j}\right)_{B}+\left(v_{j}\right)_{B}\right| \leq\left|v_{j}(x)-\left(v_{j}\right)_{B}\right|+\left|\left(v_{j}\right)_{B}\right| .
$$

By the $(1,1)$-Poincaré inequality in a ball $B,[5$, Section 7.8$]$, there exists a constant $C(n)$ such that

$$
\begin{aligned}
\left|\left(v_{j}\right)_{B}\right| & =\left(v_{j}\right)_{B}=\int_{B} v_{j}(x) d x \leq f_{B}\left|u(x)-u_{B}\right| d x \\
& \leq C(n)|B|^{\frac{1}{n}} f_{B}|\nabla u(x)| d x \leq C(n)|B|^{\frac{1}{n}-1} .
\end{aligned}
$$

We continue to estimate the right hand side of inequality (4.2)

$$
\begin{aligned}
& \int_{D} H\left(\left|u(x)-u_{B}\right|\right) d x \\
& \quad \leq \sum_{j \in \mathbb{Z}} \int_{\left\{x \in D:\left|v_{j}(x)-\left(v_{j}\right)_{B}\right|+C|B|^{-1} \geq 2^{j}\right\}} H\left(2^{j+2}\right) d x \\
& \quad \leq \sum_{j \in \mathbb{Z}} \int_{\left\{x \in D:\left|v_{j}(x)-\left(v_{j}\right)_{B}\right| \geq 2^{j-1}\right\}} H\left(2^{j+2}\right) d x+\sum_{2^{j-1} \leq C(n)|B|^{\frac{1}{n}-1}} \int_{D} H\left(2^{j+2}\right) d x \\
& \quad \leq \sum_{j \in \mathbb{Z}} \int_{\left\{x \in D:\left|v_{j}(x)-\left(v_{j}\right)_{B}\right| \geq 2^{j-1}\right\}} H\left(2^{j+2}\right) d x+\sum_{j=-\infty}^{j_{0}} \int_{D} H\left(2^{j+2}\right) d x,
\end{aligned}
$$

where $j_{0}=\left\lceil\log \left(C(n)|B|^{\frac{1}{n}-1}\right)\right\rceil$.

Assume first that diam $(D)$ is so large that $j_{0} \leq-2$. When $t<1$, then $\psi\left(t^{-1 / n}\right)=$ $\varphi(1) t^{-1 / n}$ by (2.1) and thus

$$
H^{-1}(t)=\frac{1}{\psi\left(t^{-1 / n}\right)^{n-1}}=\varphi(1)^{1-n} t^{\frac{n-1}{n}} .
$$

Thus for $t<1$ we obtain that $H(t) \approx t^{\frac{n}{n-1}}$. This yields that

$$
\begin{aligned}
\sum_{j=-\infty}^{j_{0}} \int_{D} H\left(2^{j+2}\right) d x & \approx|D| \sum_{j=-\infty}^{\left\lceil\log \left(C|B|^{\frac{1}{n}-1}\right)\right\rceil} 2^{\frac{n(j+2)}{n-1}} \leq C|D| 2^{\frac{n}{n-1} \cdot\left\lceil\log \left(C|B|^{\frac{1}{n}-1}\right)\right\rceil} \\
& \leq C|D||B|^{\frac{n}{n-1}\left(\frac{1}{n}-1\right)}=C|D||B|^{-1} \\
& \leq C \frac{\operatorname{diam}(D)^{n}}{\left(\psi\left(\frac{1}{4} \operatorname{diam}(D)\right) / c_{J}\right)^{n}}
\end{aligned}
$$


This constant does not blow up when $\operatorname{diam}(D) \rightarrow \infty$ :

$$
\frac{\operatorname{diam}(D)^{n}}{\left(\psi\left(\frac{1}{4} \operatorname{diam}(D)\right) / c_{J}\right)^{n}} \rightarrow \frac{4^{n} c_{J}^{n}}{\varphi(1)^{n}} \quad \text { as } \quad \operatorname{diam}(D) \rightarrow \infty
$$

Assume then that $\operatorname{diam}(D)$ is small. This yields that for every $j_{0} \in \mathbb{Z}$ the sum $\sum_{j=-2}^{j_{0}} H\left(2^{j+2}\right)$ is finite and depends on

$$
j_{0} \approx \log \left(C(n) \operatorname{dist}\left(x_{0}, \partial D\right)^{1-n}\right) \leq \log \left(C\left(n, c_{J}\right) \psi\left(\frac{1}{4} \operatorname{diam}(D)\right)^{1-n}\right) .
$$

We obtain

$$
\sum_{j=-\infty}^{j_{0}} \int_{D} H\left(2^{j+2}\right) d x \leq \sum_{j=-\infty}^{-2} \int_{D} H\left(2^{j+2}\right)+\sum_{j=-2}^{j_{0}} H\left(2^{j+2}\right)<\infty
$$

Then, we will find an upper bound for the sum

$$
\sum_{j \in \mathbb{Z}} \int_{\left\{x \in D:\left|v_{j}(x)-\left(v_{j}\right)_{B}\right| \geq 2^{j-1}\right\}} H\left(2^{j+2}\right) d x
$$

Since $\left\|\nabla v_{j}\right\|_{L^{1}(D)} \leq\|\nabla u\|_{L^{1}(D)} \leq 1$, Corollary 3.9 yields that

$$
\begin{aligned}
& \sum_{j \in \mathbb{Z}} \int_{\left\{x \in D:\left|v_{j}(x)-\left(v_{j}\right)_{B}\right| \geq 2^{j-1}\right\}} H\left(2^{j+2}\right) d x \\
& \quad=\sum_{j \in \mathbb{Z}} \int_{\left\{x \in D: H\left(\left|v_{j}(x)-\left(v_{j}\right)_{B}\right|\right) \geq H\left(2^{j-1}\right)\right\}} H\left(2^{j+2}\right) d x \\
& \quad \leq \sum_{j \in \mathbb{Z}} \int_{\left\{x \in D: C M\left|\nabla v_{j}\right|(x) \geq H\left(2^{j-1}\right)\right\}} H\left(2^{j+2}\right) d x .
\end{aligned}
$$

We choose for every $x \in\left\{x \in D: C M\left|\nabla v_{j}\right|(x) \geq H\left(2^{j-2}\right)\right\}$ a ball $B\left(x, r_{x}\right)$, centered at $x$ and with radius $r_{x}$ depending on $x$, such that

$$
C f_{B\left(x, r_{x}\right)}\left|\nabla v_{j}(y)\right| d y \geq \frac{1}{2} H\left(2^{j-1}\right)
$$

with the understanding that $\left|\nabla v_{j}\right|$ is zero outside $D$. By the Besicovitch covering theorem (or the 5-covering theorem) we obtain a subcovering $\left\{B_{k}\right\}_{k=1}^{\infty}$ so that we may estimate by the $\Delta_{2}$-conditionof $H$ 


$$
\begin{aligned}
& \sum_{j \in \mathbb{Z}} \int_{\left\{x \in D:\left|v_{j}(x)-\left(v_{j}\right)_{B}\right| \geq 2^{j-1}\right\}} H\left(2^{j+2}\right) d x \leq \sum_{j \in \mathbb{Z}} \sum_{k=1}^{\infty} \int_{B_{k}} H\left(2^{j+2}\right) d x \\
& \leq \sum_{j \in \mathbb{Z}} \sum_{k=1}^{\infty}\left|B_{k}\right| H\left(2^{j+2}\right) \leq \sum_{j \in \mathbb{Z}} \sum_{k=1}^{\infty} C\left|B_{k}\right| \frac{H\left(2^{j+2}\right)}{H\left(2^{j-1}\right)} \int_{B_{k}}\left|\nabla v_{j}(y)\right| d y \\
& \quad \leq C \sum_{j \in \mathbb{Z}} \int_{D}\left|\nabla v_{j}(y)\right| d y .
\end{aligned}
$$

Let $E_{j}=\left\{x \in D: 2^{j}<\left|u(x)-u_{B}\right| \leq 2^{j+1}\right\}$. Since $\left|\nabla v_{j}\right|$ is zero almost everywhere in $D \backslash E_{j}$ and $|\nabla u(x)|=\sum_{j}\left|\nabla v_{j}(x)\right| \chi_{E_{j}}(x)$ for almost every $x \in D$, we obtain

$$
\sum_{j \in \mathbb{Z}} \int_{\left\{x \in D:\left|v_{j}(x)-\left(v_{j}\right)_{B}\right| \geq 2^{j-1}\right\}} H\left(2^{j+2}\right) d x \leq C \int_{D}|\nabla u(y)| d y \leq C .
$$

Estimates (4.3), (4.4), (4.5) and (4.6) imply inequality (4.1).

Remark 4.3 In Theorem 4.2 the $N$-function $H$ is the best possible in a sense that it cannot be replaced by any $N$-function $K$ that satisfies the $\Delta_{2}$-condition and $\lim _{t \rightarrow \infty} \frac{K(t)}{H(t)}=\infty$.

In [7, Theorem 7.2] we have shown that the corresponding embedding in Theorem 4.2 does not hold if

$$
\lim _{t \rightarrow 0^{+}} t^{n} K\left(\frac{1}{\varphi(t)^{n-1}}\right)=\infty
$$

This is valid for this function $K$. By the definitions of $H^{-1}$ and $\psi$ we obtain that

$$
\lim _{t \rightarrow 0^{+}} t^{n} K\left(\frac{1}{\varphi(t)^{n-1}}\right)=\lim _{s \rightarrow \infty} \frac{1}{s} K\left(\frac{1}{\varphi\left(s^{-\frac{1}{n}}\right)^{n-1}}\right)=\lim _{s \rightarrow \infty} \frac{K\left(H^{-1}(s)\right)}{H\left(H^{-1}(s)\right)}=\infty
$$

and thus there does not exists a constant $c$ such that $\left\|u-u_{D}\right\|_{L^{K}(D)} \leq c\|\nabla u\|_{L^{1}(D)}$, for every $u \in L_{p}^{1}(D)$.

Remark 4.4 We refer to the detailed discussion in [6,7] for the fact that our result is optimal when $p=1$.

Next we prove our main theorem.

Proof of Theorem 1.2 The proof follows the idea of the proof of [10, Theorem 4.1]. By Theorems 4.1 and 4.2 there exists a constant $C$ such that the inequality

$$
\left\|u-u_{D_{i}}\right\|_{L^{H}\left(D_{i}\right)} \leq C\|\nabla u\|_{L^{p}\left(D_{i}\right)}
$$


holds for each $D_{i}$ and all $u \in L_{p}^{1}(D)$. The constant $C$ does not blow up when the diameter of $D_{i}$ tends to infinity. In the case $1<p<n$ this is clear. In the case $p=1$, we refer to the discussion after (4.4) in the proof of Theorem 4.2. The constant depends on $D_{1}$ but this does not cause a problem.

When $\|\nabla u\|_{L^{p}(D)} \leq 1$ inequality (4.7) yields that there exists a constant $C<\infty$ such that the inequality

$$
\int_{D_{i}} H\left(\left|u(x)-u_{D_{i}}\right|\right) d x \leq C,
$$

holds; here the constant $C$ is independent of $i$.

Let us write $u_{i}=u_{D_{i}}$. The triangle inequality yields that

$$
\left|u_{i}\right| \leq f_{D_{1}}\left|u(x)-u_{i}\right| d x+\int_{D_{1}}|u(x)| d x .
$$

Since $D_{i}$ satisfies inequality (4.7), we have $u \in L^{H}\left(D_{1}\right) \subset L^{1}\left(D_{1}\right)$ and thus the second term is finite. Again, by inequality (4.7) we obtain that

$$
\begin{aligned}
\int_{D_{1}}\left|u(x)-u_{i}\right| d x & \leq \frac{C\|1\|_{L^{H^{*}}\left(D_{1}\right)}}{\left|D_{1}\right|}\left\|u-u_{D_{i}}\right\|_{L^{H}\left(D_{1}\right)} \leq \frac{C\|1\|_{L^{H^{*}}\left(D_{1}\right)}}{\left|D_{1}\right|}\left\|u-u_{D_{i}}\right\|_{L^{H}\left(D_{i}\right)} \\
& \leq \frac{C\|1\|_{L^{H^{*}}\left(D_{1}\right)}}{\left|D_{1}\right|}\|\nabla u\|_{L^{p}\left(D_{i}\right)} \leq \frac{C\|1\|_{L^{H^{*}}\left(D_{1}\right)}}{\left|D_{1}\right|}\|\nabla u\|_{L^{p}(D)}<\infty .
\end{aligned}
$$

Thus the real number sequence $\left(u_{i}\right)$ is bounded and hence there exists a convergent subsequence $\left(u_{i_{j}}\right)$ and $b \in \mathbb{R}$ such that $u_{i_{j}} \rightarrow b$.

Since $H$ is continuous, $\lim _{j \rightarrow \infty} \chi_{D_{i_{j}}} H\left(\left|u(x)-u_{i_{j}}\right|\right)=\chi_{D} H(|u(x)-b|)$. Fatou's lemma and the modular form of (4.7) yield that

$$
\begin{aligned}
\int_{D} H(|u(x)-b|) d x & \leq \liminf _{j \rightarrow \infty} \int_{D} \chi_{D_{i_{j}}} H\left(\left|u(x)-u_{i_{j}}\right|\right) d x \\
& =\liminf _{j \rightarrow \infty} \int_{D_{i_{j}}} H\left(\left|u(x)-u_{i_{j}}\right|\right) \leq \liminf _{j \rightarrow \infty} C=C
\end{aligned}
$$

for every $u \in L_{\text {loc }}^{1}(D)$ with $\|\nabla u\|_{L^{p}(D)} \leq 1$. This yields that there exists a constant $C$ such that the inequality $\|u-b\|_{L^{H}(D)} \leq C$ holds for every $u \in L_{p}^{1}(D)$ with $\|\nabla u\|_{L^{p}(D)} \leq 1$. The claim follows by applying this inequality to the function $u /\|\nabla u\|_{L^{p}(D)}$.

Example 4.5 Let the function $\varphi$ be defined as in Theorem 1.2. The following unbounded domains satisfy the assumptions of Theorem 1.2:

(a) $\mathbb{R}^{n}, n \geq 2$.

(b) $\left\{\left(x^{\prime}, x_{n}\right) \in \mathbb{R}^{n}: x_{n} \geq 0\right.$ and $\left.\left|x^{\prime}\right|<\psi\left(x_{n}\right)\right\}$. 


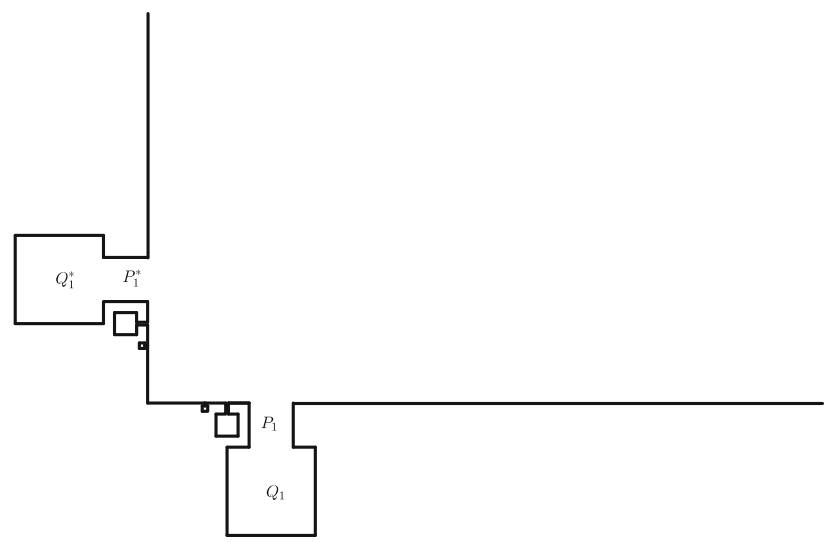

Fig. 3 Unbounded $\varphi$-cigar John domain that satisfies the assumptions of Theorem 1.2

(c) $\mathbb{R}^{2} \backslash\left(\left\{(x, \varphi(x)) \in \mathbb{R}^{2}: 0 \leq x \leq 1\right\} \cup\left\{(x,-\varphi(x)) \in \mathbb{R}^{2}: 0 \leq x \leq 1\right\}\right)$.

(d) The undounded domain $G$ constructed in Sect. 5, illustrated in Fig. 3.

\section{Lebesgue Space Cannot be a Target Space}

In this section we give an example which shows that for certain unbounded $\varphi$-cigar John domains the target space cannot be a Lebesgue space. The idea is that at near the infinity the target space should be $L^{n p /(n-p)}$ but local structure of the domain may not allow so good integrability. We assume a priori that the function $\varphi$ has the properties (1)-(5). Later on we give extra conditions to the function $\varphi$.

We construct a mushrooms-type domain. Let $\left(r_{m}\right)$ be a decreasing sequence of positive real numbers converging to zero. Let $Q_{m}, m=1,2, \ldots$, be a closed cube in $\mathbb{R}^{n}$ with side length $2 r_{m}$. Let $P_{m}, m=1,2, \ldots$, be a closed rectangle in $\mathbb{R}^{n}$ which has side length $r_{m}$ for one side and $2 \varphi\left(r_{m}\right)$ for the remaining $n-1$ sides. Let $Q$ be the first quarter of the space i.e. all coordinates of the points in $Q$ are positive. We attach $Q_{m}$ and $P_{m}$ together creating 'mushrooms' which we then attach, as pairwise disjoint sets, to the side $\left\{\left(0, x_{2}, \ldots, x_{n}\right): x_{2}, \ldots, x_{n}>0\right\}$ of $Q$ so that the distance from the mushroom to the origin is at least 1 and at most 4, see Fig. 3. We assumed that the function $\varphi$ has the properties (1)-(5), but we have to assume here also that $\varphi\left(r_{m}\right) \leq r_{m}$. We need copies of the mushrooms. By an isometric mapping we transform these mushrooms onto the side $\left\{\left(x_{1}, 0, \ldots, x_{n}\right): x_{1}, x_{3}, \ldots, x_{n}>0\right\}$ of $Q$ and denote them by $Q_{m}^{*}$ and $P_{m}^{*}$. So again the distance from the mushroom to the origin is at least 1 and at most 4 . We define

$$
G=\operatorname{int}\left(Q \cup \bigcup_{m=1}^{\infty}\left(Q_{m} \cup P_{m} \cup Q_{m}^{*} \cup P_{m}^{*}\right)\right)
$$

See Fig. 3. We omit a short calculation which shows that $G$ is a $\varphi$-cigar John domain. 
Let us define a sequence of piecewise linear continuous functions $\left(u_{k}\right)_{k=1}^{\infty}$ by setting

$$
u_{k}(x):= \begin{cases}F\left(r_{k}\right) & \text { in } Q_{k} \\ -F\left(r_{k}\right) & \text { in } Q_{k}^{*} \\ 0 & \text { in } Q,\end{cases}
$$

where the function $F$ will be given in (5.2). Then the integral average of $u_{k}$ over $G$ is zero for each $k$.

The gradient of $u_{k}$ differs from zero in $P_{m} \cup P_{m}^{*}$ only and

$$
\left|\nabla u_{k}(x)\right|=\frac{F\left(r_{m}\right)}{r_{m}} \text {, when } x \in P_{m} \cup P_{m}^{*}
$$

Note that

$$
\int_{G}\left|\nabla u_{k}(x)\right|^{p} d x=2 \int_{P_{m}}\left(\frac{F\left(r_{m}\right)}{r_{m}}\right)^{p}=2 r_{m}\left(\varphi\left(r_{m}\right)\right)^{n-1} \frac{F\left(r_{m}\right)^{p}}{r_{m}^{p}} .
$$

We require that $\int_{G}\left|\nabla u_{k}(x)\right|^{p} d x=1$. Hence, we define

$$
F\left(r_{m}\right)=\left(\frac{r_{m}^{p-1}}{2 \varphi\left(r_{m}\right)^{n-1}}\right)^{1 / p}
$$

Let $H$ be an $N$-function. Then,

$$
\begin{aligned}
& \inf _{b \in \mathbb{R}} \int_{G} H\left(\left|u_{k}(x)-b\right|\right) d x \\
& \quad \geq \inf _{b \in \mathbb{R}}\left(\left|Q_{m}\right| \cdot H\left(\left|F\left(r_{m}\right)-b\right|\right)+\left|Q_{m}^{*}\right| \cdot H\left(\left|-F\left(r_{m}\right)-b\right|\right)\right) \\
& \quad \geq r_{m}^{n} H\left(F\left(r_{m}\right)\right) .
\end{aligned}
$$

Hence, we have

$$
r_{m}^{n} H\left(F\left(r_{m}\right)\right)=r_{m}^{n} H\left(\left(\frac{r_{m}^{p-1}}{2 \varphi\left(r_{m}^{n-1}\right)}\right)^{1 / p}\right) \geq r_{m}^{n} H\left(\frac{1}{2}\left(\frac{r_{m}^{p-1}}{\varphi\left(r_{m}^{n-1}\right)}\right)^{1 / p}\right)
$$

Thus, there does not exist a positive constant $C$ such that the inequality $\inf _{b} \| u-$ $b\left\|_{L^{H}(G)} \leq C\right\| \nabla u \|_{L^{p}(G)}$ could hold for all $u$ from the appropriate space if

$$
\lim _{t \rightarrow 0^{+}} t^{n} H\left(\frac{1}{2}\left(\frac{t^{p-1}}{\varphi(t)^{n-1}}\right)^{1 / p}\right)=\infty
$$


Assume that $\lim _{t \rightarrow 0^{+}} t / \varphi(t)=\infty$. If $H(t)=t^{q}$, then we obtain that the inequality does not hold if

$$
q \geq \frac{n p}{n-p}
$$

Assume then that we have a sequence $\left(s_{j}\right)$ of positive numbers going to infinity. For each $s_{j}$ we may choose points $x(j)$ and $y(j)$ such that the balls $B\left(x(j), s_{j}\right)$ and $B\left(y(j), s_{j}\right)$ are subsets of the first quadrant and $B\left(x(j), 3 s_{j}\right) \cap B\left(y(j), 3 s_{j}\right)=\emptyset$. We define a sequence of continuous functions $\left(v_{j}\right)_{j=1}^{\infty}$ that are radially linear on $B\left(x(j), 2 s_{j}\right)$ and $B\left(y(j), 2 s_{j}\right)$ by setting

$$
v_{j}(x):= \begin{cases}s_{j}^{-\frac{n-p}{p}} & \text { in } B\left(x(j), s_{j}\right), \\ -s_{j}^{-\frac{n-p}{p}} & \text { in } B\left(y(j), s_{j}\right), \\ 0 & \text { in } G \backslash\left(B\left(x(j), 2 s_{j}\right) \cup B\left(y(j), 2 s_{j}\right)\right) .\end{cases}
$$

Now we have

$$
\int_{G}\left|\nabla v_{j}\right|^{p} d x \leq C s_{j}^{n}\left|\frac{s_{j}^{-\frac{n-p}{p}}}{s_{j}}\right|^{p} \leq C
$$

for some constant $C$. On the other hand, for any $b \in \mathbb{R}$

$$
\begin{aligned}
\int_{G} H\left(\left|v_{j}(x)-b\right|\right) d x & \geq C s_{j}^{n} H\left(\left|s_{j}^{-\frac{n-p}{p}}-b\right|\right)+C s_{j}^{n} H\left(\left|-s_{j}^{-\frac{n-p}{p}}-b\right|\right) \\
& \geq C s_{j}^{n} H\left(\left|s_{j}^{-\frac{n-p}{p}}\right|\right) .
\end{aligned}
$$

Thus, there does not exist a positive constant $C_{1}$ such that the inequality $\inf _{b} \| u-$ $b\left\|_{L^{H}(G)} \leq C_{1}\right\| \nabla u \|_{L^{p}(G)}$ could hold for all $u$ from the appropriate space if

$$
\lim _{s \rightarrow \infty} s^{n} H\left(s^{-\frac{n-p}{p}}\right)=\lim _{s \rightarrow \infty} s^{\frac{p n}{n-p}} H\left(\frac{1}{s}\right)=\infty .
$$

By choosing $H(t)=t^{q}$, we obtain that the inequality does not hold if

$$
q<\frac{n p}{n-p}
$$

If $\lim _{t \rightarrow 0^{+}} t / \varphi(t)=\infty$ and if there were an embedding with the Lebesgue space $L^{q}$ as a target space, then by (5.3) we would have $q<\frac{n p}{n-p}$ and by (5.4) we would have $q \geq \frac{n p}{n-p}$. Thus the target space cannot be a Lebesgue space. The target space can be $L^{q}$ only if $\lim _{t \rightarrow 0^{+}} t / \varphi(t)<\infty$ and in this case $q=\frac{n p}{n-p}$. Note that the limit 
$\lim _{t \rightarrow 0^{+}} t / \varphi(t)$ exists since $\varphi$ is increasing and $\varphi \geq 0$. If $\lim _{t \rightarrow 0^{+}} t / \varphi(t)=m>0$, then there exists $t_{0}>0$ such that $\frac{1}{2} m \varphi(t) \leq t \leq 2 m \varphi(t)$.

We point out that with our assumptions the case $\lim _{t \rightarrow 0^{+}} t / \varphi(t)=0$ is not possible. Namely if $\lim _{t \rightarrow 0^{+}} t / \varphi(t)=0$, then $\lim _{t \rightarrow 0^{+}} \varphi(t) / t=\infty$, and this contradicts with condition (4).

Thus we have proved the following remarks.

Remark 5.1 Let $\varphi$ satisfy (1)-(5), and assume that $\lim _{t \rightarrow 0^{+}} t / \varphi(t)=\infty$. Let $G$ be the unbounded $\varphi$-cigar John domain constructed in (5.1). Let $1 \leq p<n$. Then there do not exist numbers $q \in \mathbb{R}$ and $C \in \mathbb{R}$ such that the inequality

$$
\inf _{b \in \mathbb{R}}\|u-b\|_{L^{q}(G)} \leq C\|\nabla u\|_{L^{p}(G)}
$$

could hold for all $u \in L_{p}^{1}(G)$.

Remark 5.2 Let the function $\varphi$ satisfy conditions (1)-(5). Suppose that $\lim _{t \rightarrow 0^{+}} t / \varphi(t)$ $=m \in(0, \infty)$. Then, there exists $t_{0}>0$ such that $\varphi(t) \approx t$ for all $t \in\left(0, t_{0}\right]$. Let $G$ be the unbounded $\varphi$-cigar John domain constructed in (5.1). Assume that there exist numbers $q \in \mathbb{R}$ and $C \in \mathbb{R}$ such that the inequality

$$
\inf _{b \in \mathbb{R}}\|u-b\|_{L^{q}(G)} \leq C\|\nabla u\|_{L^{p}(G)}
$$

holds for all $u \in L_{p}^{1}(G)$. Then $q=\frac{n p}{n-p}$.

Acknowledgements Open access funding provided by University of Helsinki including Helsinki University Central Hospital. The authors would like to thank the referee for reading the manuscript carefully and for giving valuable comments.

Open Access This article is distributed under the terms of the Creative Commons Attribution 4.0 International License (http://creativecommons.org/licenses/by/4.0/), which permits unrestricted use, distribution, and reproduction in any medium, provided you give appropriate credit to the original author(s) and the source, provide a link to the Creative Commons license, and indicate if changes were made.

\section{References}

1. Bennett, C., Sharpley, R.: Interpolation of Operators. Pure and Applied Mathematics, vol. 129. Academic Press, Inc., Boston, (1988)

2. Besov, O.V., Il'in, V.P.: Natural extension of the class of regions in imbedding theorems. Math. Sb. 75(117), 483-495 (1968); translation in Math. USSR Sb. 4 (1968)

3. Edmunds, D.E., Hurri-Syrjänen, R.: Sobolev inequalities of exponential type. Isr. Math. J. 123, 61-92 (2001)

4. Fusco, N., Lions, P.L., Sbordone, C.: Sobolev imbedding theorems in borderline cases. Proc. Am. Math. Soc. 124, 561-565 (1996)

5. Gilbarg, D., Trudinger, N. S.: Elliptic Partial Differential Equations of Second Order. Reprint of the 1998 edition. Classics and Mathematics. Springer, Berlin, (2001)

6. Harjulehto, P., Hurri-Syrjänen, R.: An embedding into an Orlicz space for $L_{1}^{1}$-functions from irregular domains, complex analysis and dynamical systems VI. Part 1, contemporary mathematics. Am. Math. Soc. 653, 177-189 (2015) 
7. Harjulehto, P., Hurri-Syrjänen, R.: Pointwise estimates to the modified Riesz potential. Manuscr. Math. 156(3), 521-543 (2018)

8. Harjulehto, P., Hurri-Syrjänen, R., Kapulainen, J.: An embedding into an Orlicz space for irregular John domains, F. W. Gehring Memorial Volume, Comput. Methods Funct. Theory 14, 257-277, (2014)

9. Hedberg, L.I.: On certain convolution inequalities. Proc. Am. Math. Soc. 36, 505-510 (1972)

10. Hurri-Syrjänen, R.: Unbounded Poincaré domains. Ann. Acad. Sci. Fenn. A. I. Math. 17, 409-423 (1992)

11. Hästö, P.: The maximal operator on generalized Orlicz spaces. J. Funct. Anal. 269(12), 4038-4048 (2015)

12. John, F.: Rotation and strain. Commun. Pure Appl. Math. 14, 391-413 (1961)

13. Maz'ya, V., Poborchi, S.: Differentiable Functions on Bad Domains. World Scientific, Singapore (1997)

14. Martio, O., Sarvas, J.: Injectivity theorems in plane and space. Ann. Acad. Sci. Fenn. Ser. A. I. Math. 4(2), 383-401 (1979)

15. Näkki, R., Väisälä, J.: John disks. Expos. Math. 9, 3-43 (1991)

16. Reshetnyak, Y. G.: Integral representations of differentiable functions in domains with nonsmooth boundary (Russian), Sib. Mat. Zh, 21(1980), 108-116; translation in Sib. Math. J. 21 (1981), 833-839

17. Väisälä, J.: Quasiconformal maps of cylindrical domains. Acta Math. 162, 201-225 (1989)

18. Trudinger, N.S.: On imbeddings into Orlicz spaces and some applications. J. Math. Mech. 17, 473-483 (1967)

Publisher's Note Springer Nature remains neutral with regard to jurisdictional claims in published maps and institutional affiliations. 